\title{
Japanese Case Structure Analysis by Unsupervised Construction of a Case Frame Dictionary
}

\author{
Daisuke Kawahara, Nobuhiro Kaji and Sadao Kurohashi \\ Graduate School of Informatics, Kyoto University \\ Yoshida-Honmachi, Sakyo-ku, Kyoto, 606-8501, Japan \\ \{kawahara,kaji, kuro\}@pine.kuee.kyoto-u.ac.jp
}

\begin{abstract}
In Japanese, case structure analysis is very important to handle several troublesome characteristics of Japanese such as scrambling, omission of case components, and disappearance of case markers. However, for lack of a widecoverage case frame dictionary, it has been difficult to perform case structure analysis accurately. Although several methods to construct a case frame dictionary from analyzed corpora have been proposed, they cannot avoid data sparseness problem. 'This paper proposes an unsupervised method of constructing a case frame dictionary from an enormous raw corpus by using a robust and accurate parser. It also provides a case structure analysis method based on the constructed dictionary.
\end{abstract}

\section{Introduction}

Syntactic analysis, or parsing has been a main objective in Natural Language Processing. In case of Japanese, however, syntactic analysis cannot clarify relations between words in sentences because of several troublesome characteristics of Japanese such as scrambling, omission of case components, and disappearance of case markers. Therefore, in Japanese sentence analysis, case structure analysis is an important issue, and a case frame dictionary is necessary for the analysis.

Some research institutes have constructed Japanese case frame dictionaries manually (Ikehara et al., 1997; Information-Technology Promotion Agency, Japan, 1987). However, it is quite expensive, or almost impossible to construct a wide-coverage case frame dictionary by hand.

Others have tried to construct a case frame dictionary automatically from analyzed corpora (Utsuro et al., 1998). However, existing syntac- tically analyzed corpora are too small to learn a dictionary, since case frame information consists of relations between nouns and verbs, which multiplies to millions of combinations.

Based on sucle a consideration, we took the following unsupervised learning strategy to the Japanese case structure analysis:

1. At first, a robust and accurate parser is developed, which does not utilize a case frame dictionary,

2. a very large corpus is parsed by the parser,

3. reliable noun-verb relations are extracted from the parse results, and a case frame dictionary is constructed from them, and

4. the dictionary is utilized for case structure analysis.

\section{Characteristics of Japanese} language and necessity of case structure analysis

In Japanese, postpositions function as case markers (CMs) and a verb is final in a sentence. The basic structure of a Japanese sentence is as follows:

(1) kare ga coat wo kiru. he nominative-CM coat accusative-CM wear (he wears a coat)

A clause modifier is left to the modified noun as follows:

(2) kare ga kite-iru coat he nom-CM wear coat (the coat lie wears)

The modified noun followed by a postposition then becomes a case component of a matrix verb. The typical structure of a Japanese complex sentence is as follows: 
(3) boushi no iro wa kite-iru

hat; of color topic-marker woar

coat ni aweaseru.

coat; dative-CM harmonizc

( $\phi$ harmonizes the color of his/her hat with the coat he/she wears)

In terms of automatic analysis, the problematic characteristics of Japanese sentences can be summmarized as follows:

1. Case components are often scrambled or omitted.

2. Casc-marking postpositions disappear when case components are accompanied by topicmarkers or other special postpositions meaning 'just', 'also' and others. ex) kare wa coat mo kite-iru. he topic-1narker coat also wear (I.Ie wears a coat also)

3. A noun modified by a clause is usually a case component for the verb of the modifying clause. However, there is 10 case-marker for their relation. In case of sentence 3 , there is no case-marker for coat in rolation to kiteir'u 'wear'. Note that ni (dative-CM) of coat' ni does not show the case to kite-ir'u "woar", but to awaseru 'hammonize'.

4. Sontence 3 exhibits a typical structural ambiguity in a Japanese sentence. 'J'hat is, iro wa 'color topic-narker' possibly modifies kite-iru 'wear" or awasern 'harmonizo'.

In English, sentence structure is rather rigid, and word order (the position in relation to the verb) clearly defines cases. In Japanese, however, the problem 1 above makes word order useless, and CMs constitute the only information for detecting cases.

Nevertheless, CMs often disappear because of the problems 2 and 3, which means that simple syntactic analysis cannot clarify cases sufficiently. For example, given an input sentence:

(4) kare wa Deutsch-go mo hanasu. he topic-marker German also speak (he speaks German also)

a simple syntactic analysis just detects both kare 'he' and Deutsch-go 'German' modifies hanasu 'speak', but tells nothing about which is subject and object. 'This analysis result is not sufficient for subsequent NLP applications like Japanese to English machine translation.

'Then, what we need to do is a case structure analysis based on a case frame dictionary, or a subcat, of each verb as follows:

\begin{tabular}{|c|c|}
\hline \multicolumn{2}{|c|}{ hanasu 'speak': } \\
\hline$g a(\mathrm{nom})$ & kare 'he', hito 'porson' \\
\hline wo (acc) & eigo 'English', kotoba 'language' \\
\hline \multicolumn{2}{|c|}{ kiru 'woar': } \\
\hline$g a(110111)$ & kare 'he', hito 'person' \\
\hline wo $(\mathrm{acc})$ & fuku 'cloth', cout 'coat' \\
\hline \multicolumn{2}{|c|}{ awaseru 'harmonize': } \\
\hline$g a(\mathrm{nom})$ & kare "he', hito "person' \\
\hline wo $(\mathrm{acc})$ & iro 'color' \\
\hline$n i$ (dat) & fuku 'cloth' \\
\hline
\end{tabular}

Consultation of such a dictionary can casily find that kare 'he' is a nominative case and Deutschgo 'Geman' is an accusative case in the sentence 4.

Furthermore, a case frame dictionary can solve the problem 4 above, that is, some part of structural ambiguity in sentences. In case of sentence 3 , a proper head for iro wa 'color topic-marker' can be selected by consulting case slots of kiru 'wear' and those of awaseru 'harmonizo'

\section{Unsupervised construction of a case frame dictionary}

'I'his section explains how to construct a case frame dictionary from corpora antomatically.

As nentioned in the introduction section, it is quite expensive, or almost impossible to construct, a wide-coverage case frame dictionary by hand. In Japanese, some noun + copula works like an adjective. For example, sansei da 'positiveness + copula' can take ga case and ni case. However, such case frames are rarely covered by the existing handmade dictionaries ${ }^{1}$.

Furthermore, existing handmade dictionaries cover typical obligatory cases like ga (nominative), wo (accusative), ni (dative), but do not cover compound case markers such as ni-kianshite 'in terms of', wo-megutte 'concerning' and others.

Then, we tried to construct an example-based case frame dictionary from corpora, which de-

\footnotetext{
${ }^{1}$ Our method collects case frames not only for verbs, but also for adjectives and nouns+copula. In this paper, we use 'verb' instead of 'verl)/adjective or noun t- copula' for simplicity.
} 
Table 1: The accuracy of KNP.

\begin{tabular}{lllllllll|l}
\hline $\begin{array}{l}\text { ga } \\
\text { nom. }\end{array}$ & $\begin{array}{l}\text { acc. } \\
\text { ac. }\end{array}$ & $\begin{array}{l}\text { ni } \\
\text { dative }\end{array}$ & $\begin{array}{l}\text { kara } \\
\text { from }\end{array}$ & $\begin{array}{l}\text { made } \\
\text { to }\end{array}$ & $\begin{array}{l}\text { yori } \\
\text { from }\end{array}$ & $\begin{array}{l}\text { wa, mo } \\
\text { topic- } \\
\text { marker }\end{array}$ & $\begin{array}{l}\text { clause } \\
\text { modifying } \\
\text { verbs }\end{array}$ & $\begin{array}{l}\text { clause } \\
\text { modifying } \\
\text { nouns }\end{array}$ & 'Total \\
\hline $91.2 \%$ & $97.7 \%$ & $94.2 \%$ & $83.8 \%$ & $85.3 \%$ & $82.8 \%$ & $88.0 \%$ & $84.3 \%$ & $95.5 \%$ & $91.3 \%$ \\
\hline
\end{tabular}

scribes what kind of cases each verb has and what kind of nouns can fill a case slot. Very large syntactically analyzed corpora could be useful to construct such a dictionary. However, corpus annotation costs very much and existing analyzed corpora are too small from the view point of case frame learning. For example, in Kyoto University Corpus which consists of about 40,000 analyzed sentences of newspaper articles, very basic verbs like tetsudau 'help' or uketsukeru 'accept' appear only 10 times or 15 times respectively. It is obvious that such small data are insufficient for automatic case frame learning. That is, case frame learning must bo done from enormous unanalyzed corpora, in unsupervised way ${ }^{2}$.

\subsection{Good parser}

NLP research group at Kyoto University has been developing a robust and accurate parsing system, KNP, over the last ten years (Kurohashi and Nagao, 1994; Kurohaslii and Nagao, 1998). This parser has the following advantages:

- Japanese is an agglutinative language, and several function words (auxiliary verbs, suffixes, and postpositions) often appear together and in many cases compositionality does not hold among them. KNP treats such function words carefully and precisely.

- KNP detects scopes of coordination structures well based on their parallelism.

- KNP employs several heuristic rules to produce unique parses for the input sentences.

The accuracy of KNP is shown in Table 1, which counted whether each phrase modifies a proper head or not. The overall accuracy was around $90 \%$, and the accuracy concerning case components varies from $82 \%$ to $98 \%$.

\footnotetext{
${ }^{2}$ In English, several unsupervised methods have been proposed (Manning, 1993; Briscoe and Carroll, 1997). However, as mentioned in Section 3, automatic Japanese case analysis is much harder than English.
}

We can collect pairs of verbs and case components from the automatic analyses of large corpora by KNP.

\subsection{Coping with two problems}

The quality of automatic case frame learning could be negatively influenced by the following two problcms:

Word sense ambiguity: A verb sometimes has various usages and possibly has several case frames depending on its usages.

Structural ambiguity: KNP performs fairly well, but automatic parse results inevitably contain errors.

'The following sections explain how to solve these problems.

\subsubsection{Word sense ambiguity}

If a vorb has two or more meanings and their case frame patterns differ, wo have to disambiguate the sense of each occurrence of the verb in a corpus first, and collect case components for each sense respectively. However, unsupervised word sense disambiguation of free texts is one of the most difficult problems in NLP. At the very beginning, even the definition of word senses is open to question.

To cope with this problem, we made a very simple but useful assumption: a light verb has different case frames depending on its main case component; an ordinary verb has a unique case frame even if it has two or more meanings. For example, the case frame of the verb naru 'become' differs depending on its ni (dative) case as follows:

$$
\begin{aligned}
& \text { ․ ga byouki ni naru } \\
& \text { nom. become ill } \\
& \begin{array}{lllll}
\cdots & \text { ga } & \cdots & \text { to } & \text { tomodachi ni naru }
\end{array} \\
& \text { nom. with become a friend }
\end{aligned}
$$

In most cases, the main case components aro placed just in front of the light verbs so that the automatic parser can detect their rclations 
'Table 2: Examples of the constructed case frames.

\begin{tabular}{|c|c|c|}
\hline verlos & case markers & example noums \\
\hline $\begin{array}{l}\text { tasukcru } \\
\text { "holls" }\end{array}$ & $\begin{array}{l}g a \text { (nom) } \\
\text { wo (acc) } \\
\text { ni (dat) } \\
\text { de (op) }\end{array}$ & $\begin{array}{l}\text { lussband, person, child, staff, I, suspect, faculty, ... } \\
\text { job, shop, farmwork, preparation, election, move, ... } \\
\text { son, friend, ambassador, member, thank, holiday, ... } \\
\text { volunteer, affair, office, reward, house, headquarters, ... }\end{array}$ \\
\hline $\begin{array}{l}\text { yomu } \\
\text { "roadl" }\end{array}$ & $\begin{array}{l}y a(\text { nom }) \\
\text { wo (acc) } \\
\text { ni (dati) } \\
\text { de (op) }\end{array}$ & $\begin{array}{l}\text { person, I, child, adult, parent, toacher, ... } \\
\text { newspaper, book, maggazine, artiche, novel, letter, ... } \\
\text { child, person, daughter, teacher, student, reader, ... } \\
\text { newspaper, book, magevine, library, classroom, bathroom, ... }\end{array}$ \\
\hline
\end{tabular}

reliably. 'Therefore, as for five major and tronblesome light verbs (sur'b 'to', naru 'bocomo', aru 'is ..', iu 'say', nai 'not'), their case frames are distinguished depending on their loft neigh. bouring case components. For other vorbs, we assume a unique case frame.

\subsubsection{Structural ambiguity}

As shown in 'Table 1, KNP detects hoads of case components in fairly ligh acenary. However, in order to collect much reliable datar, wo disarded modifier-head relations in the antomatically parsed corpora in the following cases:

- When CMs of case components disappear because of topic markers on others.

- When the verb is followed by a cantsative anxiliary or a passive anxiliary, the case pattorn is chamged and the trace in KNI' is not: so reliable.

Based on the conditions alove, case compoments of each verb are collected from the parsed corjpora, and the collected data are considered as case frames of verbs. However, if the firequency of a CM is very low compared to other CMs, it might be collected because of parse errors. So, we set the threshold for the CM frequency as $2 \sqrt{m f}$, where $m f$ means the frequency of the most found CM. If the frequency of a CM is less than the threshold, it is discarded. For example, suppose the most frequent CM for a verb is wo, 100 times, and the frequency of $n i \mathrm{CM}$ for the verb is 16 , ni $\mathrm{CM}$ is discarded (since it is less than the threshold, 20).

\subsection{Constructed case frame dictionary}

We applied the above procedure to Mainichi Newspaper Corpus (7 years, $3,600,000$ sentonces). From the corpus, case firames of 23,497 verbs are constucted; the average number of case slots of a verb is 2.8 ; the average number of example nouns in a case slot is 33.6. Table 2 shows examples of constructed case frames.

Although the constructed data look appropriate in most cases, it is loard to evaluate a clictionary statically. In the next section, we use the dictionary in caso structure analysis and evaluate the analysis result, which also inmplies an evaluation of the dictionary itsolf.

\section{Case structure analysis using the constructed case frame dictionary}

\subsection{Matching of an input sentence and a case frame}

T'he basic procedure in case structure analysis is to match an input sentence with a case frame, as shown in figure 1.

'The matching of case components in an input, and case slots in a case frame is done on the following conditions:

1. When a cass component has a CM, it must be assigned to the case slot with the same CM.

2. When a case component does not have a CM, it can be assigned to the $g a, w o$, or $n i$ CM slot.

3. Only one case component can be assigned to a case slot (unique caso assigmment constraint).

The conditions above may produce multiple matching patterns, and to select the proper one among them, nouns of case components are compared with examples in case slots of the dictionary. 

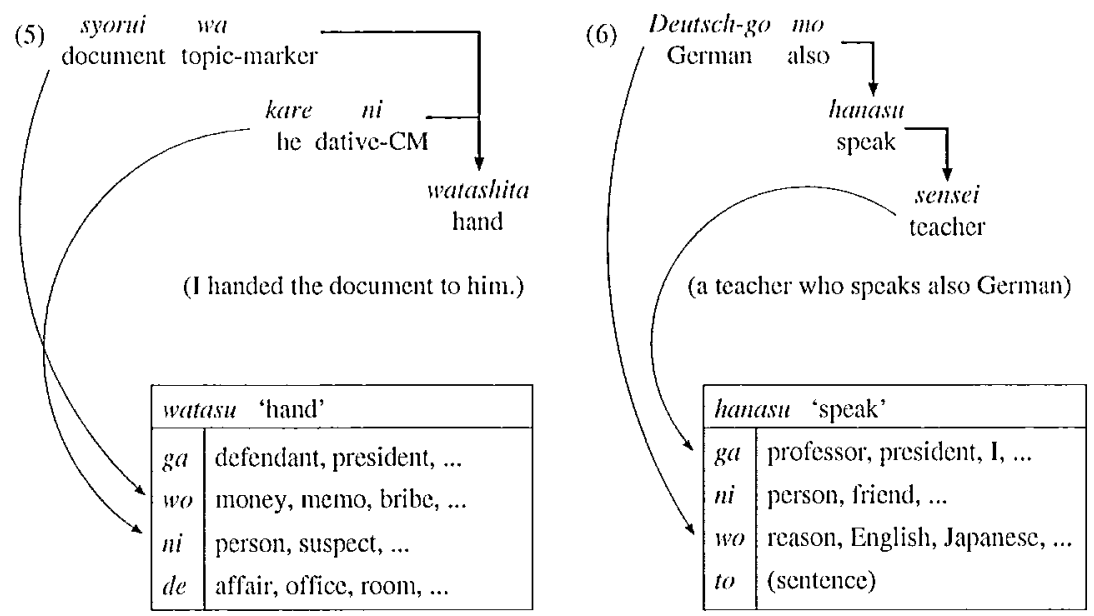

Figure 1: Matching of an input sentence and a casc frame.

Even though a $3,600,000$ sentences corpus was used for learning, cxamples in case slots are still sparse, and an input noun mostly does not match exactly an example in the dictionary. Then, a thesaurus is employed to solve this problem.

In our experiments, NI'I Semantic Feature Dictionary (Ikehara et al., 1997) is employed as a thesaurus. Suppose we calculate the similarity between $w_{1}$ and $w_{2}$, their depth is $d_{1}$ and $d_{2}$ in the thesaurus, and the depth of their lowest (most specific) common node is $d_{c}$, the similarity score between them is calculated as follows:

$$
\operatorname{sim}\left(w_{1}, w_{2}\right)=\left(d_{c} \times 2\right) /\left(d_{1}+d_{2}\right) .
$$

If $w_{1}$ and $w_{2}$ are in the same node of the thesaturtus, the similarity is 1.0 , the maximum score based on this criteria. If $w_{1}$ and $w_{2}$ are identical, the similarity is 1.0 , of course.

The score of case assignment is the best similarity between the input noun and examples in the case slots. The score of a matching pattorn is the sum of scores of case assignments in it. If two or more patterns meet the above conditions, one which has the best score is selected as a final result.

In the case of sentence 5 in Figure 1, kare ni 'he dative-CM' is assigned to the $n i$ case slot. Then, syorui wa 'document topic-marker' can be assigned to the ga or wo case slot. By calculating similarity between syorui and ga-slot examples and wo-slot examples, it is considered to be assigned to the wo slot.

In case of sentence 6 , none of the case components has a CM. Based on similarity calculation,
Deutsch-go is assigned to wo, sensei is assigned to ga.

\subsection{Parsing with case structure analysis}

A complex sentence which contains a clausal modifier exlibits a typical structural ambiguity of Japanese; case components left to a verb of a clausal modifier, $V_{c}$, possibly modify $V_{c}$ or a matrix verb $V_{m}$.

For example, in sentence 3, iro wa 'color topic-marker' possibly modifies kite-iru 'wear' or awaseru 'harmonize'.

KNP, a rule-based parser, handles this type of ambiguity as follows. If a case component is followed by a comma, it is treated as modifying $V_{m}$; if not, it is treated as modifying $V_{c}$. Although this heuristic rule usually explains real data vory well, sentence 3 will be analyzed incorrectly.

Parsing which utilizes a case frame dictionary can consider which is a proper head, $V_{c}$ or $V_{m}$, for an ambiguous case component by comparing examples in the case slots of $V_{c}$ and $V_{m}$. Such a consideration must be done considering what other case components modify $V_{c}$ and $V_{m}$, since the assigned case slot of a case component might differ depending on the candidate structure of the sentence due to the unique caso assignment constraint.

Therefore, it is necessary to expand the structural ambiguity and consider all the possible structures for an input. So, we calculate the matching score of all pairs of case components and verbs in all possible structures of the sentence, and select the best structure based on the 

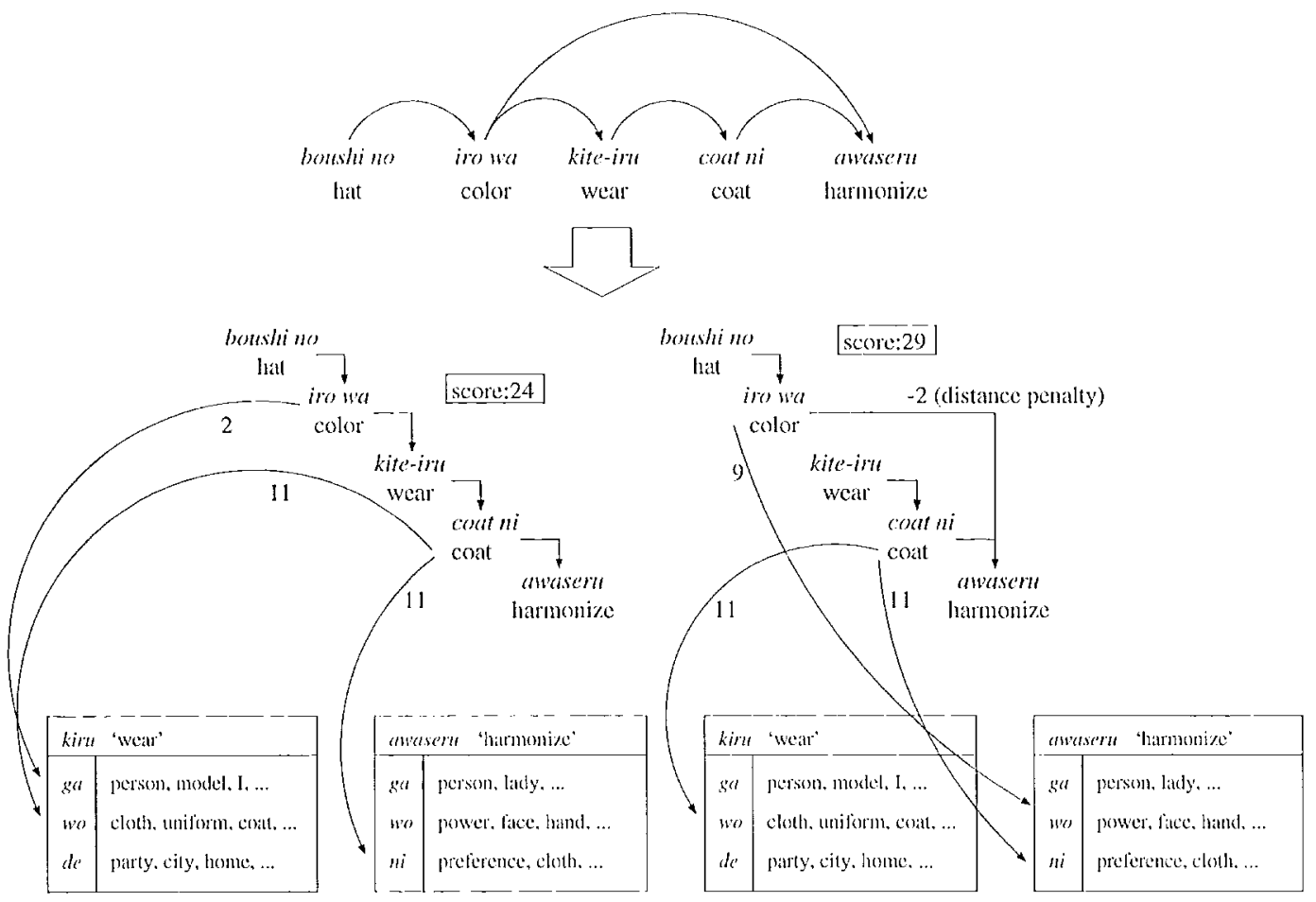

Figue 2: Parsing with case structure analysis.

sum of the matching scores in it.

Since the heuristic rule employed in $\mathrm{KNP}^{\mathrm{P}}$ is actually very useful, we incorporate it, that is, penalty score is inposed to the modifier-head relation depend ling on the distance between a moctifice and a heacl. If a modifier is not followed by a comma, the penalty score, $0,-2,-4,-6, \ldots$ is imposed when a modifier modifies the first (noaresti), second, third, fourth, ... verbs in a sentence respectively; if with a comma, the penalty score, $-2,0,-2,-4, \ldots$ is imposed.

For example, sentence 3 was analyzed by our method as shown in Figure 2. Since the sinilanity score between iro 'color' and the wo-slot of aumaser'u 'harmonize' is much larger thian that between iro 'color' and the ga-slot of kiru 'wear', the correct structure of the sentence was detected (the right-hand parse of Figure 2). Note that, furthermore, both the case of iro in relation to awaseru 'harmonize', and the case of coat in relation to kite-iru 'wear' were detected correctly.

Structural ambiguities often cause a combinatorial explosion when a sentence is long. However, by detecting the scopes of coordinate structures beforehand, which often appear in long
'Jable 3: 'The accuracy of case detection.

\begin{tabular}{c|c|c|c}
\hline & $\begin{array}{l}\text { correct } \\
\text { calse } \\
\text { detection }\end{array}$ & $\begin{array}{l}\text { incorrect } \\
\text { calse } \\
\text { detection }\end{array}$ & $\begin{array}{l}\text { Parsing } \\
\text { oror }\end{array}$ \\
\hline topic-marker & 82 & 13 & 5 \\
clausal modifier & 73 & 1.8 & 9
\end{tabular}

sentences, we can reasonably linnit the possible structures of the sentence.

The average andysis speed of the experiments described in the next section was about 50 sentences/min. The time-out of one min. wass only employed to 7 out of 4,272 test sentences.

\subsection{Experiments and discussion}

We used 4,272 sentences of Kyoto University corpus as a test set. We parsed them by our now method (Figure 3 shows several examples) and checked two points: case detection of ambiguous caso components and syntactic analysis.

First, we randomly selected ambiguous case components: 100 topic-marked casc components and 100 case components modified by clausal 


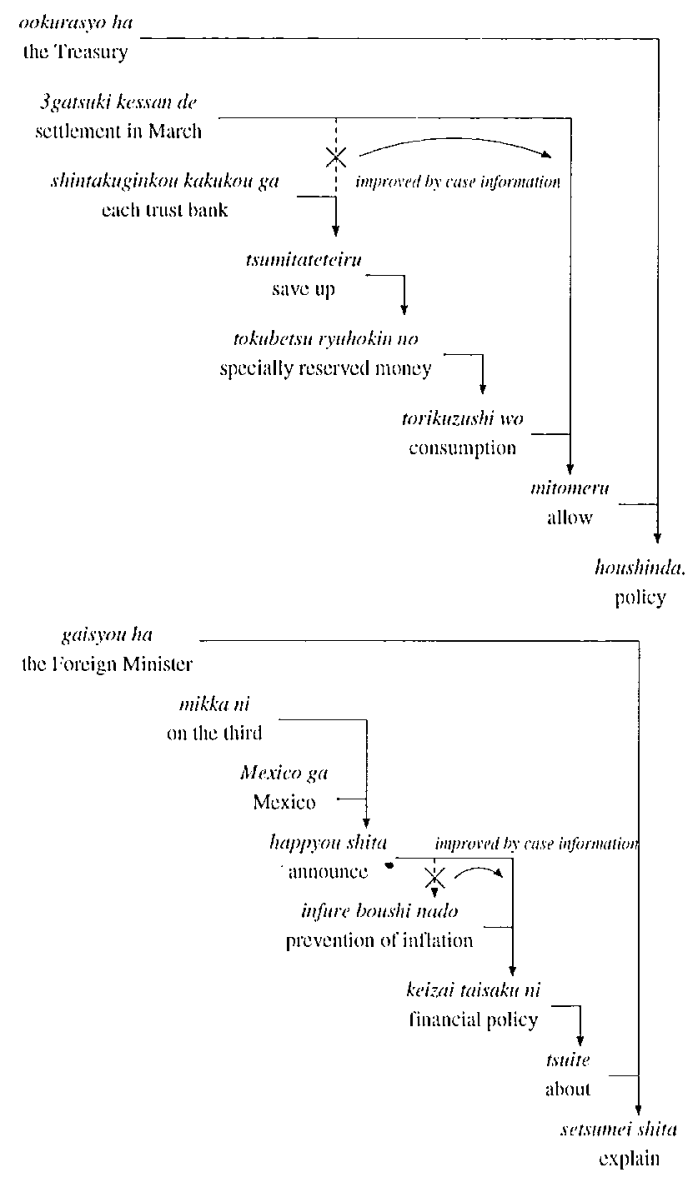

Figure 3: Examples of the analysis results.

modifiers, and checked whether their cases were correctly detected or not. As shown in Table 3, the accuracy of the analysis was fairly good: that for topic-markers was $82 \%$ and that for clausal modifiers was $73 \%$.

Then, we compared the parse results of our method with those of the original KNP. As a result, 565 modifier-head relations differed; in 260 cases, our method was correct and the original $\mathrm{KNP}$ was incorrect (by considering the structures in the Kyoto University Corpus as a golden standard); in 224 cases, vice versa. That is, our method was superior to KNP by 36 cases, and increased the overall accuracy from $89.8 \%$ to $89.9 \%$. Since the heuristic rule used in KNP is very strong, the improvement was not big. The improvement of the accuracy, though small, is valuable, because the accuracy around $90 \%$ seems close to the ceiling of this task.

\section{Conclusion}

We proposed an unsupervised construction method of a case frame dictionary. We obtained a large case frame dictionary, which consists of 23,497 verbs. Using this dictionary, we can detect ambiguous case components accurately. Also since our method employs unsupervised dictionary learning, it can be easily scaled up.

\section{References}

Ted Briscoe and John Carroll. 1997. Automatic extraction of subcategorization from corpora. In Proceedings of ANLP-9\%.

Satoru Ikehara, Masahiro Miyazaki, Satoshi Shirai, Akio Yokoo, Hiromi Nakaiwa, Kentarou Ogura, and Yoshifumi Oyama Yoshiliko Hayashi, editors. 1997. Japanese Lexicon. Iwanami Publishing.

Information-Technology Promotion Agency, Japan. 1987. Japanese Verbs: A Guide to the IPA Lexicon of Basic Japanese Verbs.

S. Kurohashi and M. Nagao. 1994. A syntactic analysis method of long japanese sentences based on the detection of conjunctive structures. Computational Linguistics, 20(4).

S. Kurohashi and M. Nagao. 1998. Building a japanese parsed corpus while improving the parsing system. In Proceedings of the First International Conference on Language Resources 8 Evaluation, pages 719-724.

Christopher D. Manning. 1993. Automatic acquisition of a large subcategorization dictionary from corpora. In Proceedings of $A C L_{-}-93$.

Takchito Utsuro, Takashi Miyata, and Yuji Matsumoto. 1998. General-to-specific model selection for subcategorization preference. In Proceedings of the 17th International Conference on Computational Linguistics and the 36th Annual Mecting of the Association for Computational Linguistics. 